Pour détecter les planètes extrasolaires, l'utilisation du phénomène de microlentille gravitationnelle présente le grand avantage par rapport aux autres méthodes d'être la seule sensible aux planètes de faible masse situées

à relativement grande distance de leur étoile - telle une Neptune ou une Terre en orbite à une dizaine d'unités astronomiques (UA). Nous présentons ici la découverte, grâce à cette technique, de la première planète tellurique, OGLE-2005-

BLG-390Lb, une cousine glacée de la Terre environ cing fois plus massive, évoluant sur une orbite de 2,6 UA autour d'une étoile naine rouge. définis dans le lexique.

\title{
Découverte de la première planète extrasolaire tellurique
}

Jean-Philippe Beaulieu ${ }^{1,2,3}$ (beaulieu@iap.fr) et Arnaud Cassan ${ }^{1,2,4}$

1) Collaboration PLANET - 2) Programme HOLMES

3) Institut d'Astrophysique de Paris, CNRS, Université Pierre et Marie Curie UMR 7095, 98 bis, boulevard Arago, 75014 Paris

4) Astronomisches Rechen-Institut (ARI), Zentrum für Astronomie, Universität Heidelberg, Mönchhofstr. 12-14, 69120 Heidelberg, Allemagne

La fin du vingtième siècle a vu se transformer en réalité l'un des plus grands défis de l'Astronomie moderne : découvrir des planètes autour d'étoiles autres que le Soleil.Voilà déjà 12 ans qu'à l'Observatoire de Haute Provence, Michel Mayor et Didier Queloz ont détecté une planète de masse moitié moindre que Jupiter, en orbite autour de l'étoile 51 de la constellation de Pégase (51 Peg b) [1]. Grâce aux progrès instrumentaux et à la mise au point de nouvelles méthodes de détection, c'est tout un nouveau champ d'investigation qui s'est ouvert [2,3], et près de 300 planètes qu'on appelle désormais " extrasolaires " sont connues à ce jour [4]. Une vaste compétition internationale s'est engagée, avec de grandes ambitions pour le futur. L'intérêt scientifique lié à la quête de ces planètes est en effet multiple : quelle est leur abondance et comment interpréter la grande diversité de leurs caractéristiques ? Quels sont les processus physico-chimiques qui ont présidé à leur formation ? Quels liens ont-elles entretenu avec leur disque protoplanétaire`, berceau probable de leurs premiers stades d'évolution?

Un panorama des planètes et systèmes planétaires connus à ce jour fait en premier lieu apparaitre une très riche diversité de leurs caractéristiques. Des "Jupiter " chauds telle 51 Peg $b$ - planètes gazeuses, massives et extrêmement proches de leur étoile - aux planètes rocheuses, froides et distantes comme OGLE2005-BLG-390Lb, c'est toute une gamme de conditions physiques qui s'offre aux astronomes. Les premières détections ont tout d'abord dévoilé un certain nombre de planètes géantes gazeuses de la famille de Jupiter, pour progressivement révéler l'existence de planètes de plus en plus petites. L'une des questions majeures est de savoir quelle place notre système solaire tient parmi ses homologues extrasolaires, comment il s'intègre ou se distingue des autres.

Actuellement, les modèles de formation des systèmes planétaires favorisent les scénarios d'accrétion autour d'un cour : les planétésimaux ${ }^{\star}$ s'accumulent pour former des cœurs planétaires qui ensuite accrètent du gaz de la nébuleuse primitive [5]. Une prédiction de ce scénario dans le cas des étoiles naines $\mathrm{M}\left(\mathrm{de} 0,1\right.$ à $\left.0,5 \mathrm{M}_{\odot}{ }^{\star}\right)$ est que les planètes de faible masse ${ }^{\star}$ (comme Neptune ou la Terre) se forment en quelques millions d'années, à des distances comprises entre 1 et 10 UA. Les planètes plus massives (telle Jupiter) se forment, quant à elles, bien plus difficilement, car leur formation nécessite souvent un laps de temps (environ 10 millions d'années) plus grand que la durée de vie du disque protoplanétaire, et il ne reste plus de gaz pour les entourer d'une atmosphère.

Deux méthodes parmi les plus populaires sont la technique de mesure de vitesses radiales et celle des transits. Une planète en orbite autour d'une étoile induit une variation de la position de cette dernière (par rapport au centre de gravité étoile-planète) : l'oscillation de la distance aux observateurs terrestres se traduit par un déplacement Doppler de ses raies spectrales, alternativement vers le rouge et le bleu, que l'on peut mesurer par spectroscopie de précision - c'est la méthode dite des vitesses radiales. Dans certaines configurations, une planète va effectuer un passage - ou transit - devant le disque stellaire et provoquer une diminution apparente mesurable de la luminosité de l'étoile : c'est la méthode dite des transits. Autrefois limitée à la détection de planètes massives et proches de leur étoile, la méthode des vitesses radiales permet à présent de détecter des "Jupiter " sur une vaste gamme d'orbites (de 0,02 à 5 UA»), et des « Neptune " sur des orbites très courtes (inférieures à $0,2 \mathrm{UA}$ ). Les récentes mesures avec le spectrographe francosuisse HARPS montrent que les planètes de quelques masses terrestres sur orbite courte sont désormais accessibles. Le satellite COROT, lancé avec succès en décembre 2006, devrait aussi détecter des Neptune chauds, sur des orbites de 0,02 à 0,4 UA.

Si ces deux méthodes ont démontré leur grande efficacité, elles présentent cependant un maximum de sensibilité pour des planètes géantes sur des orbites plutôt proches de leur étoile. 


\section{Lexique}

\section{Unité astronomique (UA)}

Distance entre la Terre et le Soleil (149,6 millions de km). Cette unité est utilisée pour mesurer les distances dans les systèmes planétaires.

\section{Parsec (pc)}

Le parsec est défini comme étant la distance à laquelle une unité astronomique (UA) sous-tend un angle d'une seconde d'arc. Un parsec vaut 3,26 années-lumière, soit environ $3,1 \times 10^{16} \mathrm{~m}$. Cette unité est utilisée pour mesurer les distances à plus grande échelle. Le centre de notre galaxie est situé à 8 kpc.

\section{Milliseconde d'arc (mas)}

Un degré est subdivisé en 60 minutes d'arc, elles-mêmes divisées en 60 secondes d'arc. La milliseconde d'arc vaut $10^{-9}$ radians, soit $2 \mathrm{~m}$ à la distance de la Lune.

\section{Rayon d'Einstein (RE)}

C'est une quantité ne dépendant que de la masse de la lentille et des distances entre la source, la lentille et l'observateur. Dans le cas d'un alignement parfait sourcelentille-observateur, l'image de la source est un anneau de rayon $R E$, de diamètre angulaire $~ 1$ mas (voir l'encadré de l'article de Y. Mellier, dans Reflets de la physique $n^{\circ} 1$, p. 9).

\section{Masses}

La masse du Soleil est de $1,99 \times 10^{30} \mathrm{~kg}$, et définit l'unité $\left(M_{\odot}\right)$ utilisée par les astronomes. Deux autres unités, utilisées régulièrement, sont la masse de Jupiter $\left(M_{\jmath}\right)$ et la masse de la Terre $\left(M_{T}\right)$. On a les relations suivantes $1 M_{\odot}=1050 M_{J}$ et $M_{J}=318 M_{T}$ La masse de Neptune vaut $17 \mathrm{M}_{\mathrm{T}}$.

\section{Planétésimaux}

Corps rocheux préplanétaires.

\section{Disque protoplanétaire}

Les étoiles se forment à partir d'un nuage de gaz et de poussière en effondrement. La matière se condense dans un disque autour de la protoétoile en formation, et au sein de ce disque se forment des grumeaux qui vont devenir des planètes.

\section{Bulbe et disque galactiques}

Dans l'univers, les étoiles sont regroupées dans de vastes ensembles, appelés galaxies. La Voie lactée est une galaxie spirale, formée d'un disque dans lequel se distribuent les étoiles le long des bras spiraux, et d'un bulbe composé de vieilles étoiles au centre.

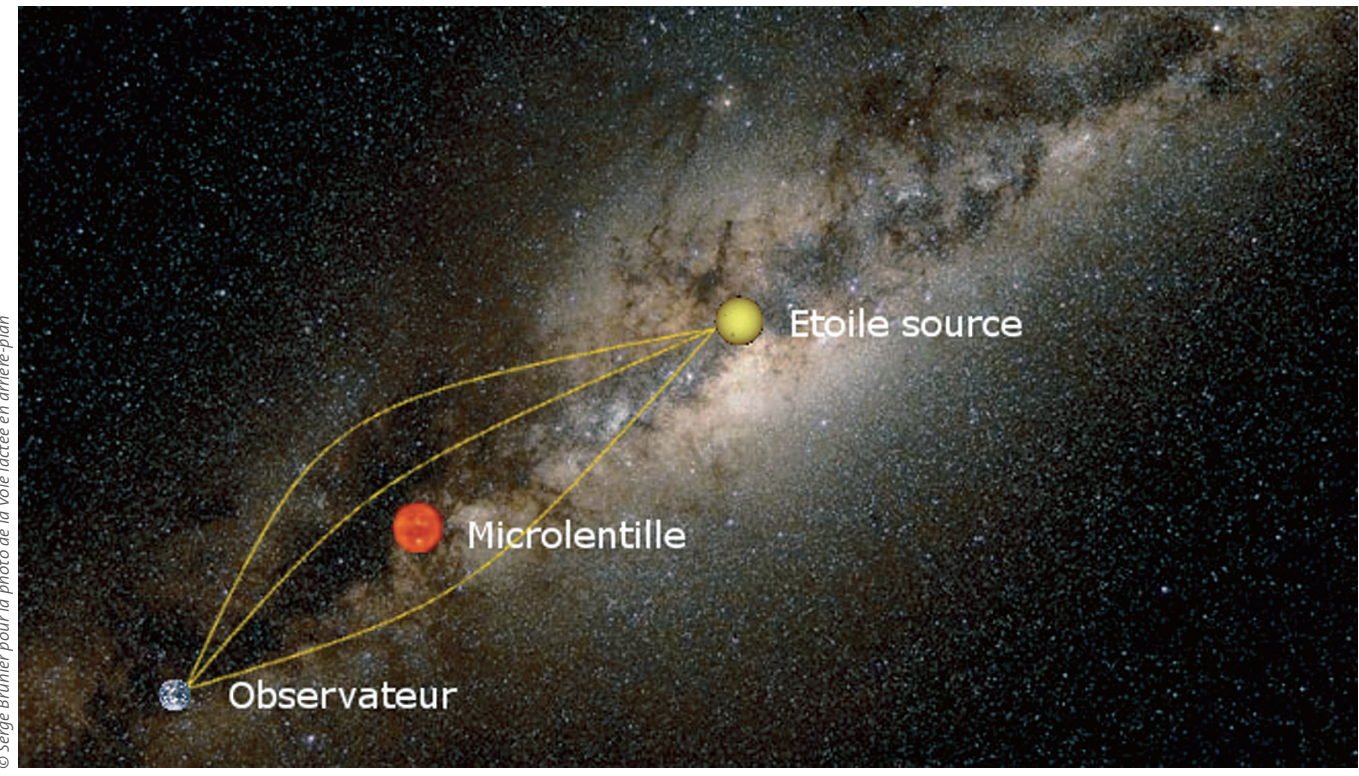

Figure 1 : Description du phénomène de microlentille sur une photo de la Voie lactée (notre galaxie). Un phénomène de microlentille gravitationnelle galactique (aussi appelé "événement de microlentille ") a lieu lorsqu'une étoile lointaine, souvent située dans la partie centrale de la Voie lactée (le bulbe), passe très près de la ligne de visée d'une étoile d'avant-plan. Les rayons lumineux de l'étoile d'arrière-plan subissent alors une déviation, conformément aux lois de la relativité générale. Lorsque la microlentille est une étoile isolée, le flux lumineux de l'étoile source suit une courbe en cloche symétrique, très caractéristique du phénomène (en bas à gauche de la figure 2).

Prises à part, elles offrent ainsi une vision quelque peu biaisée, et le recours à des méthodes explorant des domaines d'orbite et de masse complémentaires est indispensable. Cet aspect est fondamental, en particulier dans le cas des planètes en orbite autour d'étoiles de faible masse, comme nous allons le voir.

Nous utilisons une autre méthode pour chercher des planètes extrasolaires, le phénomène de lentille gravitationnelle. Sa spécificité est d'offrir une sensibilité maximum pour des planètes sur des orbites de quelques UA, et non pas au plus proche de leur étoile. Elle présente ce grand avantage par rapport aux méthodes concurrentes d'être la seule méthode sensible à des exoplanètes sur des orbites de 1 à 10 UA, et pour un domaine de masses s'étageant de la Terre à Jupiter.

\section{Détecter une planète par effet de microlentille}

Durant un événement de microlentille, le flux lumineux d'une étoile "source » distante est temporairement amplifié par le passage d'un objet massif - la "lentille » - très proche de la ligne de visée vers l'étoile source (une fraction de milliseconde d'arc ${ }^{\star}$ ) (fig. 1). L'amplification dépend directement de la distance angulaire entre source et lentille. Elle est exprimée dans une unité particulière, celle du « rayon d'Einstein "^, unité qui dépend de la masse de la lentille et des distances entre l'observateur, la source et la lentille. Pour une étoile source du centre de notre galaxie (le bulbe ${ }^{\star}$ ) et une étoile lentille du disque ${ }^{\star}$, le rayon d'Einstein est de l'ordre de 2 UA ; quant à la durée caractéristique du phénomène de microlentille, il est de l'ordre de 10 à 30 jours le plus souvent, et presque toujours compris entre 2 et 150 jours. Une planète en orbite autour de l'étoile lentille génère en général des « caustiques gravitationnelles », lignes imaginaires en projection sur le plan du ciel où l'amplification devient formellement infinie : si une étoile source traverse une de ces caustiques, un signal additionnel indiquera à coup sûr la présence de la planète autour de la lentille par une augmentation (ou une diminution) brève et brutale du flux amplifié en provenance de l'étoile source (fig. 2). La durée de cette perturbation planétaire est proportionnelle à la racine carrée de sa masse ; elle peut durer quelques heures pour une planète comme la Terre, jusqu'à quelques jours pour une planète plus massive (telle Jupiter).

Déterminer la nature de la lentille ayant généré la variation de luminosité observée, autrement dit le problème inverse, est malheureusement relativement complexe, parce que les modèles sont hautement non linéaires et l'espace des paramètres à explorer, vaste et parsemé de minima locaux. Le recours à des algorithmes performants (algorithmes génétiques ou méthodes de type "Markov Chain Monte-Carlo ") permet de surpasser la plupart de ces difficultés, mais ils sont extrêmement coûteux en temps et puissance de calcul. À l'issue de cette phase de modélisation, il est possible de déterminer le rapport de masse entre l'étoile et la planète, ainsi que leur séparation instantanée projetée sur le plan du ciel, en unité de rayon d'Einstein. Dans la plupart des 


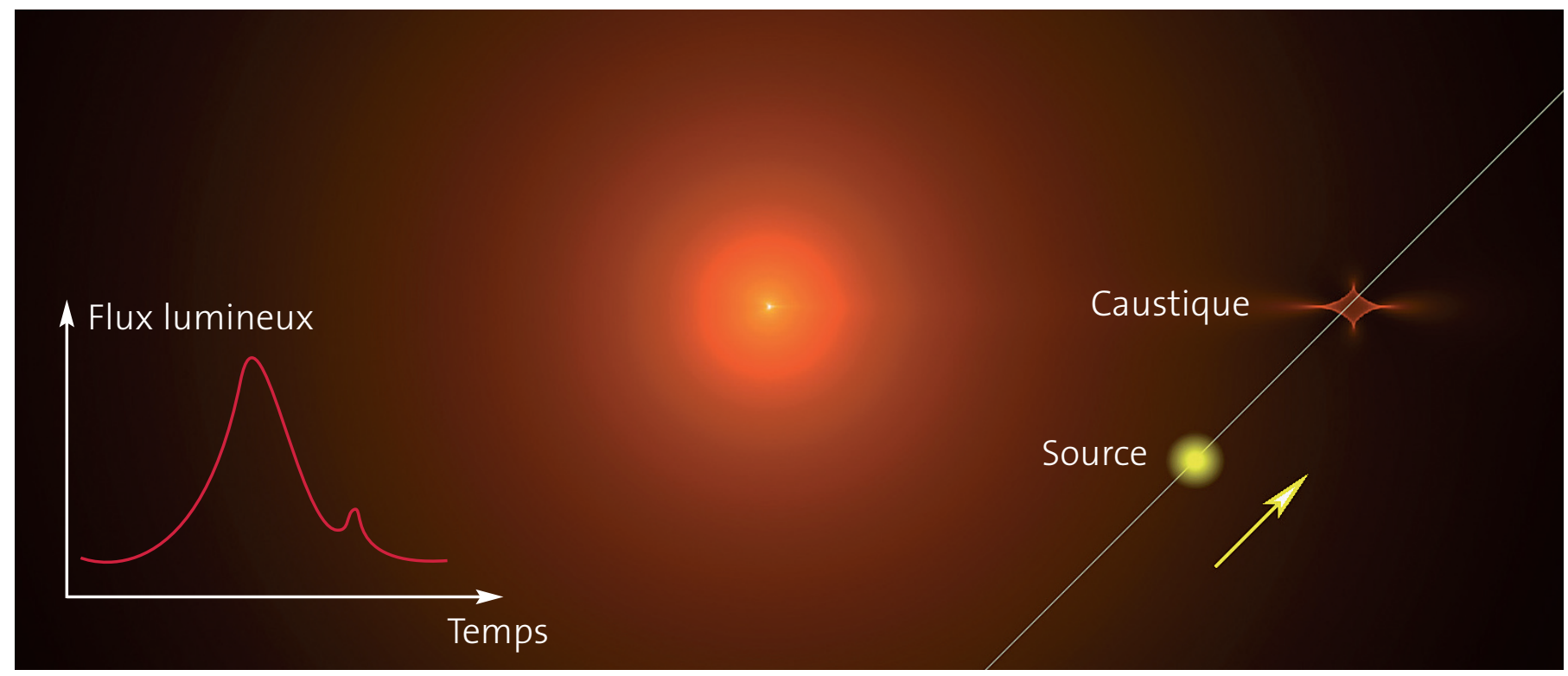

Figure 2 : Les microlentilles binaires sont composées de deux corps gravitationnellement liés, par exemple un système étoile-planète, et possèdent des propriétés remarquables. Sur cette figure, l'amplification du flux de la source en fonction de sa position est représentée par une carte projetée sur le ciel. L'amplification est d'autant plus grande que la couleur est claire à la position de source considérée ; les lignes brillantes sont des caustiques gravitationnelles, lieux d'accumulation de lumière comme en optique classique (ces caustiques sont situées entre les deux composantes de la microlentille). Au cours de son trajet (rectilinéaire en première approximation), la source (disque jaune) subit une amplification qui trace en fonction du temps la courbe présentée dans l'insert en bas à gauche de la figure. Le pic principal d'amplification est atteint lorsque le trajet de la source passe au plus près du centre de masse du système de lentilles. Quant au pic secondaire, il est produit lors de la traversée par la source de la petite caustique en forme de diamant. C'est ce dernier pic qui révèle la présence de la planète.

cas, une dernière étape faisant intervenir un modèle de notre galaxie se révèle nécessaire pour obtenir les paramètres du système planétaire dans des unités physiques habituelles. Ces paramètres sont alors estimés par une analyse de type bayesienne, qui fournit les densités de probabilités pour chaque paramètre du système.

\section{Stratégie d'observation}

L'observation de phénomènes de microlentille pose un défi observationnel. Il consiste à suivre un grand nombre de tels événements pour détecter et mesurer, sur des échelles de temps de quelques heures, des perturbations dans leur courbe de lumière dues à la présence de planètes. Des télescopes dédiés à des opérations de grands relevés (OGLE, MOA, voir glossaire, et autrefois EROS et MACHO) détectent aujourd'hui environ 600 microlentilles par an, et rendent publiques leurs positions dans le ciel.

Notre collaboration PlANET/RoBONET (voir glossaire) utilise alors un réseau de huit télescopes, de $0,6 \mathrm{~m}$ à $2 \mathrm{~m}$ de diamètre et équipés de caméras CCD, pour observer en continu, 24 heures sur 24 , un certain nombre de microlentilles. La stratégie d'observation est optimisée et coordonnée pour maximiser les chances de détecter en temps réel le signal caractéristique engendré par la présence d'une planète.

\section{La découverte d'une planète de 5,5 masses terrestres}

Le 11 juillet 2005, la collaboration OGLE annonça sa $390^{\mathrm{e}}$ découverte d'une microlentille pour l'année en cours. Elle fut inscrite sur la liste de la vingtaine d'objets suivis par le réseau Planet/RoboNet. Le maximum d'amplification fut atteint le 31 juillet. Nous avons continué à l'observer, et le 10 août les observateurs au télescope EsO danois au Chili, notèrent une déviation de $6 \%$ par rapport à une lentille simple ; une autre mesure une heure plus tard révélait cette fois une déviation de $12 \%$. La collaboration OGLE publia alors ses données en ligne, et ses points de mesure venaient confirmer l'observation qu'une anomalie était en cours. Le télescope de Perth en Australie commença ses observations, mais ne put transmettre ses données. Du côté de l'Afrique du Sud, le ciel était couvert et, quand les observations recommencèrent au Chili, l'anomalie était terminée. Finalement, quand les données de Perth furent disponibles, elles confirmèrent qu'une anomalie de 10 heures avec une amplitude de 30\% avait été observée (fig. 3). Deux mesures complémentaires de la collaboration MOA furent disponibles quelques jours plus tard, renforçant les preuves de l'observation d'un signal.

\section{Glossaire : programmes de recherche de planètes extrasolaires}

Collaboration PLANET (Probing Lensing Anomalies NETwork)

Programme international initié en 1995, regroupant une trentaine d'astronomes de 10 pays et utilisant 5 télescopes répartis dans l'hémisphère sud (Perth en Australie, Boyden et Sutherland en Afrique du Sud, ESO-La Silla au Chili, Hobart en Tasmanie (Australie)), pour effectuer une observation quasi continue des événements de microlentilles dans le bulbe galactique. En 2005, PLANET a joint ses forces au réseau RobotNet.

RobotNet

Réseau britannique de trois télescopes (Liverpool, Hawaï et Australie)

HOLMES (Hunting cOol Low Mass Extrasolar planetS)

Programme blanc 2006 de l'Agence nationale pour la Recherche.

HARPS (High Accuracy Radial velocity for Planetary Search)

Consortium franco-suisse piloté par l'Observatoire de Genève, utilisant un spectrographe pour mesure précise des vitesses radiales, situé au télescope ESO de La Silla (Chili)

OGLE (Optical Gravitational Lensing Experiment)

Collaboration américano-polonaise de recherche de la matière noire.

MOA (Microlensing Observations in Astrophysics)

Collaboration Nouvelle-Zélande-Japon 
Figure 3 : Observations des variations de luminosité d'une étoile galactique dues à la microlentille OGLE-2005-BLG-390. Dans l'insert en haut à gauche, les observations d'OGLE entre 2001 et 2005. La figure principale représente la variation de la luminosité en juillet-août 2005 ; les différentes couleurs correspondent à divers télescopes ayant suivi ce phénomène. La ligne noire correspond à la meilleure description théorique du phénomène. Vers le 11 août, une anomalie est observée, qui est agrandie dans l'insert en haut à droite.

\section{En savoir plus...}

$1 \cdot$ M. Mayor et D. Queloz, Nature 378 (1995) 355.

2 F. Casoli et T. Encrenaz, Bulletin de la SFP Hors série BUP 875 (2005) 117.

$3 \cdot F$. Selsis, Bulletin de la SFP 154 (2006) 5.

4 - Voir l'encyclopédie interactive sur les planètes extrasolaires http://exoplanet.eu/

5 - G. Laughlin et al., ApJ 612 (2004) L73.

6 -J.P. Beaulieu, D.P. Bennett, P. Fouqué et al., Nature 439 (2006) 437.

7 D. Ehrenreich et al., ApJ 651 (2006) 535

8-A. Cassan et al., 2007, Proceedings of the IAU Symposium n 249 (2008), p. 119.

$9 \cdot$ B.S. Gaudi et al., arXiv:0802.1920v1, février 2008.

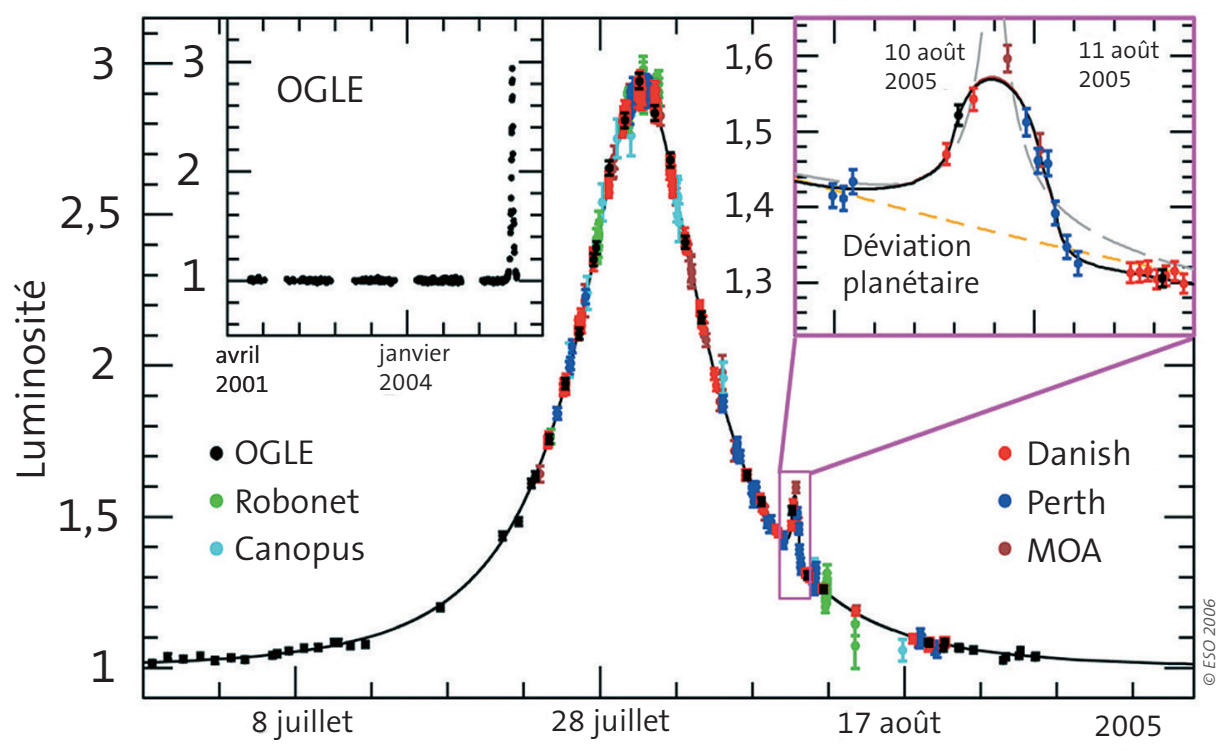

Une activité frénétique de modélisation débuta dans l'enthousiasme et, très rapidement, il fut clair qu'une petite planète venait d'être détectée. L'analyse fut relativement aisée, et donna un rapport de masse avec son étoile de $q=(7,6 \pm 0,7) 10^{-5}$, et une séparation projetée $\mathrm{d}=1,61 \pm 0,008$ (en unité du rayon d'Einstein). Nous avons alors réalisé une analyse bayesienne pour déterminer les distributions de probabilité des paramètres du système planétaire. Les valeurs médianes conduisent à une étoile de masse $0,22^{+0,21}{ }_{-0,11} \mathrm{M}_{\odot}$, située à une distance de $6,6 \pm 1,1 \mathrm{kpc}^{\star}$ dans le bulbe galactique.

La planète, OGLE-2005-BLG-390Lb, de masse égale à $5,5^{+5,5}-2,7$ Terres, est sur une orbite de $2,6^{+1,5}{ }_{-0,6}$ UA [6]. Il s'écoula à peine un mois entre la découverte de la planète et la soumission de l'article pour publication.

\section{Esquisse d'un portrait d'OGLE-2005-BLG-390Lb}

À partir des informations disponibles, certaines caractéristiques du système planétaire peuvent être inférées. Tout d'abord, cette planète est en orbite autour d'une naine de type $M$, petite étoile froide et peu lumineuse. L'énergie reçue à sa surface étant de $0,1 \mathrm{~W} / \mathrm{m}^{2}$ (15000 fois moins que sur Terre), c'est certainement un monde glacial présentant une température de surface proche de $40 \mathrm{~K}$, ce qui est comparable à Pluton. C'est aussi une planète tellurique, composée essentiellement de roches et de corps volatils, et elle pourrait être de même nature que la Terre ou, en supposant une fraction beaucoup plus importante d'eau dans sa composition, appartenir à la famille supposée des planètes-océans. Son cœur et son manteau rocheux produiraient une importante quantité de chaleur, dont l'origine serait la désintégration radioactive des isotopes de l'uranium, du thorium et du potassium ; elle pourrait être alors en mesure de maintenir un océan d'eau liquide sous une épaisse couche de glace durant plusieurs milliards d'années. Cependant, comme la planète est localisée dans le bulbe de notre galaxie, son âge probable avoisine dix milliards d'années (soit deux fois l'âge de notre Soleil), ce qui en fait un système très vieux. Le taux de chauffage venant de son manteau rocheux ayant décru au cours du temps, elle est donc vraisemblablement entièrement gelée aujourd'hui [7].

Les modèles ont pu montrer que pour les petites planètes très froides comme OgLe-2005-BLG-390Lb, l'existence d'un océan liquide sous une épaisse couche de glace serait possible durant plusieurs milliards d'années. On remarquera l'analogie avec certains satellites du système solaire, tels Europe et Ganymède, voire même certains objets de la ceinture de Kuiper, où l'on pense possible l'existence d'un océan liquide sous une couche de glace.

\section{Ces planètes sont-elles très répandues?}

Nous avons effectué un premier bilan de l'efficacité de l'application de la technique de microlentilles à la détection de planètes extrasolaires, basé sur les données du programme Planet/Robonet [8]. À ce jour, quatre planètes ont été détectées par cette méthode, deux géantes gazeuses et deux petites planètes glacées, pour lesquelles nous avons des efficacités de détection d'environ $50 \%$ et $2 \%$ respectivement. Plus récemment, une équipe menée par l'Ohio State University a mis en évidence par microlentille gravitationnelle un nouveau système planétaire qui possède un couple de planètes Jupiter-Saturne [9] ; il n'est pas exclu qu'autour de cette étoile existent aussi d'autres 
planètes intérieures bien moins massives et rocheuses, telles Mars ou la Terre, à l'instar de notre système solaire. Bien qu'il soit encore trop tôt pour tirer des conclusions définitives, le fait que le nombre de détections soit le même pour les petites planètes que pour les plus massives (et ce malgré une efficacité de détection bien plus faible pour les planètes peu massives) semble montrer que les petites planètes rocheuses seraient bien plus abondantes que les géantes gazeuses.

Les observations continuent, et d'ici environ quatre ans, nous espérons disposer d'une bonne estimation de l'abondance des petites planètes telluriques, avant le démarrage de programmes d'encore plus grande envergure pour détecter des "Terre " dans des conditions physicochimiques favorables à l'éclosion de la vie [3].

Cet article fut rédigé à l'invitation d'Alain Milsztajn, physicien au CEA, membre du comité de rédaction de Reflets de la Physique. Alain Milsztajn était un des piliers de la collaboration Eros (Expérience de Recherche d'Objets Sombres) qui traquait la matière noire dans le halo de notre galaxie par effet de microlentille gravitationnelle. Dans ses dernières semaines, il mettait la dernière touche à l'article donnant les résultats finaux d'ERos combinant 14 ans de données, et il nous aidait à rédiger ce petit article. Il nous a toujours encouragé dans nos travaux à la recherche des planètes par effet de microlentille sur les 12 dernières années. Enthousiaste, sympathique, d'une rigueur scientifique à toute épreuve, Alain était aussi le copain qui chantait de la musique baroque dans la coupole sous les étoiles, pendant que le télescope d'Eros traquait des microlentilles... 